\title{
Prevalência de hiperidrose primária e fatores associados em uma capital do nordeste do Brasil: estudo baseado em população
}

\author{
Prevalence of primary hyperhidrosis and associated factors in a capital city in northeastern \\ Brazil: a population-based study
}
Prevalencia de hiperhidrosis primaria y factores asociados en una ciudad capital del noreste de Brasil: un estudio de base poblacional

Sonia Oliveira Lima ${ }^{1 *}$, Carla Viviane Freitas de Jesus ${ }^{1}$, Yasmim Anayr Costa Ferrari ${ }^{1}$, Rafael Silva Santos $^{1}$, Edna Santos Dias ${ }^{1}$, Lorenna Oliveira Menezes ${ }^{1}$, Rute Nascimento da Silva ${ }^{1}$, Renata Lima Batalha de Andrade ${ }^{1}$, Felipe Mendes de Andrade de Carvalho ${ }^{1}$, Vanessa Rocha de Santana ${ }^{1}$.

\section{RESUMO}

Objetivo: Avaliar a prevalência de hiperidrose primária (HP) e fatores associados em uma amostra populacional de uma capital do nordeste brasileiro. Métodos: Estudo transversal com amostras populacionais de cinco bairros aleatórios de uma capital do nordeste brasileiro. Questionário validado "Diagnóstico da hiperidrose primária". Resultados: De 1330 indivíduos, 34,2\% homens e 65,8\% mulheres. A prevalência de HP foi de $17 \%$ entre os homens, $14,9 \%$ entre mulheres e teve valor global de $15,9 \%$. A idade média dos entrevistados foi de 25,9 \pm 10,2 anos. A idade de início ocorreu principalmente entre 0 e 19 anos de idade. Tanto o suor bilateral como a piora dos sintomas com estresse apresentaram valores significativos $(p<0,05)$. A transpiração foi principalmente bilateral, pelo menos uma vez por semana e agravada com o estresse $(p<0,05)$. Conclusão: A prevalência de HP na população amostrada foi de $15,9 \%$, semelhante em ambos os sexos e, na maioria dos casos, bilateral. O início dos sintomas ocorreu principalmente durante a infância e adolescência, com implicações negativas nas atividades diárias, e os sintomas primários de hiperidrose são exacerbados com o estresse.

Palavras-chave: Hiperidrose, Epidemiologia, Características da população.

\begin{abstract}
Objective: To assess the prevalence of primary hyperhidrosis $(\mathrm{PH})$ and associated factors in a population sample from a capital city in northeastern Brazil. Methods: Cross-sectional study with population samples from five random neighborhoods of a capital of northeastern Brazil. Validated questionnaire "Diagnosis of primary hyperhidrosis". Results: Of 1330 individuals, $34.2 \%$ men and $65.8 \%$ women. The prevalence of $\mathrm{PH}$ was $17 \%$ among men, $14.9 \%$ among women and had an overall value of $15.9 \%$. The average age of the interviewees was $25.9 \pm 10.2$ years. The age of onset occurred mainly between 0 and 19 years of age. Both bilateral sweating and worsening symptoms with stress showed significant values $(p<0.05)$. Sweating was mainly bilateral, at least once a week and worsened by stress $(p<0.05)$. Conclusion: The prevalence of PH in the sample population was $15.9 \%$, similar in both sexes and, in most cases, bilateral. The onset of symptoms occurred mainly during childhood and adolescence, with negative implications for daily activities, and the primary symptoms of hyperhidrosis are exacerbated by stress.
\end{abstract}

Key words: Hyperhidrosis, Epidemiology, Population characteristics.

1 Universidade Tiradentes (UNIT), Aracaju - SE. *E-mail: sonialima.cirurgia@gmail.com 


\section{RESUMEN}

Objetivo: Evaluar la prevalencia de hiperhidrosis primaria (HP) y factores asociados en una muestra de población de una ciudad capital del noreste de Brasil. Métodos: Estudio transversal con muestras de población de cinco barrios aleatorios de una capital del noreste de Brasil. Cuestionario validado "Diagnóstico de hiperhidrosis primaria". Resultados: De 1330 individuos, 34.2\% hombres y 65.8\% mujeres. La prevalencia de HP fue del $17 \%$ entre los hombres, el $14,9 \%$ entre las mujeres y tuvo un valor general del $15,9 \%$. La edad promedio de los entrevistados fue de $25.9 \pm 10.2$ años. La edad de inicio se produjo principalmente entre los 0 y los 19 años. Tanto la sudoración bilateral como el empeoramiento de los síntomas con estrés mostraron valores significativos $(p<0.05)$. La sudoración fue principalmente bilateral, al menos una vez por semana y empeoró por el estrés $(p<0.05)$. Conclusión: La prevalencia de HP en la población de la muestra fue de $15.9 \%$, similar en ambos sexos y, en la mayoría de los casos, bilateral. La aparición de los síntomas se produjo principalmente durante la infancia y la adolescencia, con implicaciones negativas para las actividades diarias, y los síntomas principales de la hiperhidrosis se ven exacerbados por el estrés.

Palabras clave: Hiperhidrosis, Epidemiología, Caracteristicas de la poblacion.

\section{INTRODUÇÃO}

A hiperidrose é uma condição em que a produção de suor excede os requisitos para a regulação da temperatura corporal e é classificada como primária ou secundária. A hiperidrose primária (HP) é idiopática e a hiperidrose secundária tem associações com muitas entidades, incluindo distúrbios endócrinos, terapia medicamentosa, câncer, neuropatias (LAKRAJ AA, et al., 2013). A apresentação clínica quando se trata do local primário de transpiração na HP, tende a estar relacionada às áreas com alta concentração de glândulas sudoríparas, como palmas, solas e axilas e a sudação geralmente é simétrica (WALLING HW, 2011).

A incidência é semelhante em ambos os sexos e o aparecimento de HP palmar e plantar ocorre frequentemente durante a infância e adolescência. A HP também é pensada ser agravada por estímulos emocionais e tem uma história familiar positiva (STRUTTON DR, et al., 2004; BELLET JS, 2010; LIMA SO, et al., 2019). É uma doença crônica que pode causar desconforto considerável no paciente, comprometendo sua vida em ambientes sociais, afetivos e profissionais, contribuindo para transtornos de ansiedade (SCHNEIER FR, et al., 2012; BRAGANÇA GMG, et al., 2014).

A falta de conhecimento social sobre a doença, a presença de sintomas depressivos, como sentimentos de vergonha e falta de autoconfiança, está presente nos portadores de HP (GROSS KM, et al., 2014). É importante diagnosticar esta condição, pois o tratamento é relativamente simples. As opções de tratamento não cirúrgico variam desde tratamento tópico, iontoforese a tratamento sistêmico (HORNBERGER J, et al., 2004; CERFOLIO RJ, et al., 2011; WOLOSKER N, et al., 2011; HONG C-HH, et al., 2012; WOLOSKER N, et al., 2012; LIMA SO, et al., 2013). O tratamento cirúrgico consiste em ablação axilar das glândulas sudoríparas e/ou simpatectomia endoscópica torácica ou lombar, esses dois fornecem níveis de satisfação variando de 72\% a 94\% entre os pacientes (ADAR R, et al., 1977; TU Y-R, et al., 2007; HEIDEMANN E, et al., 2013; DELAPLACE M, et al., 2015).

Muitos dos estudos de HP são direcionados ao diagnóstico e ao tratamento, e apenas alguns deles demonstraram interesse em descrever a população afetada por esta doença crônica, cuja prevalência é variável indo de $0,6 \%$ a $18 \%$ entre as poucas pesquisas publicadas (ADAR R, et al., 1997; LIMA SO, et al., 2019). O objetivo do presente estudo é obter dados sobre prevalência e fatores associados aos sintomas de HP em uma amostra de população de uma capital na região nordeste do Brasil.

\section{MÉTODOS}

Os indivíduos estudados foram de cinco bairros escolhidos aleatoriamente de uma capital localizada no nordeste do Brasil. A população consistiu de residentes da cidade que possuíam registro de identidade da cidade avaliada. A prevalência de $16,6 \%$ para hiperidrose primária foi estimada de um estudo anterior (AUGUSTIN M, et al., 2013), usando a fórmula da população finita e considerando uma precisão absoluta de 2\% e um nível de significância de 5\%, 1330 participantes foram entrevistados (MIOT HA, 2011). 
A construção da amostra de estratificação foi feita com base na proporção da soma do número total de pessoas em cinco bairros em relação ao número total de pessoas em cada bairro como resultado da amostragem necessária. A partir desta estratificação, encontrou-se 0 número de pessoas a serem entrevistadas em cada distrito.

A coleta de dados foi realizada através de entrevistas individuais com o objetivo de evitar interpretações erradas das questões. O questionário, estruturado com perguntas de escolha múltipla, já foi validado e aborda aspectos como gênero, idade, etnia, critérios diagnósticos para HP idiopática e características relacionadas aos sintomas (FIORELLI RKA, et al., 2011).

Os entrevistadores eram estudantes de medicina treinados para a técnica de entrevista, que receberam instruções de um profissional médico sobre o conteúdo específico de cada pergunta. As visitas domiciliares foram realizadas com a assistência e monitoramento de um funcionário de saúde pública do governo local.

Todos os indivíduos foram fisicamente e mentalmente capazes de responder ao questionário e assinaram um consentimento informado. Participantes menores de idade foram incluídos com a devida autorização de seus representantes legais.

Usando as definições de idade da Organização Mundial da Saúde, o estudo considerou crianças de 0 a 9 anos e adolescentes de 10 a 19 anos. Os critérios de exclusão da amostra consistiram naqueles que apresentavam características clínicas ou diagnóstico prévio de condições predisponentes à hiperidrose secundária, como infecciosa, mediada por drogas, endócrina, neurológica, maligna (VORKAMP T, et al., 2010).

A prevalência foi calculada pelo número de indivíduos com o desfecho (hiperidrose primária) dividido pelo número total de indivíduos na amostra. O teste Qui-quadrado foi utilizado para as associações das variáveis com o resultado. Todas as análises estatísticas foram realizadas com o SPSS $\AA$ v20.0. A análise descritiva foi realizada utilizando frequências absolutas e relativas no caso de variáveis categóricas e por medidas de tendência e variabilidade central no caso de variáveis numéricas. Então, a análise inferencial das características dos pacientes com hiperidrose foi realizada por meio de intervalo de confiança de $95 \%$. O valor de significância foi inferior a 0,05. A coleta de dados foi aprovada pela Comissão Nacional de Ética em Pesquisa (050613R2).

\section{RESULTADOS}

Foi entrevistada uma quantidade total de 1330 voluntários; 34,2\% (455) eram do sexo masculino e 65,8\% (875) eram do sexo feminino. A prevalência geral de hiperidrose primária foi de 15,9\% (212). Os entrevistados diagnosticados com HP não apresentaram evidência clínica de hiperidrose secundária. A prevalência de gênero foi de $17 \%$ entre os homens e $14,9 \%$ entre as mulheres. A idade média dos entrevistados com diagnóstico clínico de HP foi de 25,9 $\pm 10,2$ anos. O início dos sintomas ocorreu HP mais frequentemente durante a infância e adolescência (82,5\%), como mostrado na Tabela 1.

Tabela 1 - Idade de início da transpiração excessiva nos entrevistados diagnosticados com hiperidrose primária em uma amostra da população de uma cidade no nordeste do Brasil de 2014 ( $n=212)$.

\begin{tabular}{cc}
\hline Idade de início & $\mathbf{n}(\%)$ \\
\hline $0-5$ anos & $33(15,5)$ \\
$6-10$ anos & $27(12,7)$ \\
$11-15$ anos & $49(23,2)$ \\
$16-19$ anos & $66(31,1)$ \\
$20-25$ anos & $23(10,8)$ \\
26 ou + anos & $14(6,7)$ \\
\hline
\end{tabular}

Fonte: Lima SO, et al., 2020.

As associações de HP com gênero $(p=0,479)$ e etnia $(p>0,05)$, não foram estatisticamente significantes. $O$ intervalo de frequência e de confiança (IC) de fatores associados com os sintomas em portadores de hiperhidrose primária é mostrada na Tabela 2. 
Tabela 2 - Frequência e intervalo de confiança $(\mathrm{Cl})$ de fatores associados aos sintomas em portadores de hiperidrose primária em uma amostra populacional de uma cidade no nordeste do Brasil, 2014.

\begin{tabular}{cccc}
\hline Fatores associados com Hiperidrose Primária & $\mathbf{n}(\%)(\mathbf{S i m})$ & $\mathbf{I C ~ 9 5 \% ~ M i n ~}$ & Max \\
\hline Sudorese bilateral e simétrica & $133(73)^{*}$ & 66 & 79 \\
História familiar & $70(38)$ & 32 & 45 \\
Presença de sintomas pelo menos uma vez por semana & $110(60)^{*}$ & 53 & 67 \\
Cessação dos sintomas durante o sono & $71(39)$ & 32 & 46 \\
Interferência da temperatura & $76(42)$ & 35 & 49 \\
Prejuízo nas atividades diárias & $56(31)$ & 24 & 38 \\
Agravação dos sintomas com estresse & $140(77)^{*}$ & 70 & 82 \\
\hline
\end{tabular}

Legenda: ${ }^{*} p<0,05$. Fonte: Lima SO, et al., 2020.

Hiperidrose primária, ainda que seja uma doença benigna, pode levar a limitações significativas no estilo de vida pessoal e profissional. Por outro lado, os dados epidemiológicos sobre HP ao redor do mundo são variáveis e escassos como pode-se observar na Tabela 3.

Tabela 3 - Prevalência mundial da Hiperidrose Primária de 1997 a 2019.

\begin{tabular}{cccc}
\hline País & Autor & Ano de publicação & Prevalência de HP \\
\hline Israel & ADAR R, et al. & 1997 & $0,6-1,0 \%$ \\
EUA & STRUTTON DR, et al. & 2004 & $2,9 \%$ \\
China & TU Y-R, et al. & 2007 & $4,59 \%$ \\
Brasil & FENILI R, et al. & 2009 & $9 \%$ \\
Brasil & WESTPHAL FL, et al. & 2011 & $5,5 \%$ \\
Japão & FUJIMOTO T, et al. & 2013 & $12,76 \%$ \\
Alemanha & AUGUSTIN M, et al. & 2013 & $16,6 \%$ \\
Polônia & STEFANIAK T, et al. & 2013 & $16,7 \%$ \\
Brasil & LIMA SO, et al. & 2015 & $14,76 \%$ \\
Brasil & CAMPOS JJG, et al. & 2019 & $11,11 \%$ \\
Brasil & LIMA SO, et al. & 2019 & $18 \%$ \\
\hline
\end{tabular}

Fonte: Lima SO, et al., 2020.

Mesmo que o presente estudo não tenha utilizado a Escala de Severidade da Hiperidrose HDSS, pudemos inferir que 56 indivíduos dos 212 que relataram HP, afirmaram que a doença causa prejuízo nas atividades diárias. Isso sugere que eles sofrem de suor dificilmente tolerável que frequentemente interfere nas atividades diárias, sendo, consequentemente, classificados como HDSS 3 ou HDSS 4. Esses dois tipos de HP podem estar associados a uma morbidade considerável, incluindo lesões cutâneas e infecções secundárias da pele, podendo ficar edemaciada, macerada ou até rachada (BELLET JS, et al., 2010; DEL SORBO F, et al., 2011). Os scores da escala estão descritos na Tabela 4.

Tabela 4 - Escala de Severidade da Hiperidrose - Hyperhidrosis Disease Severity Scale (HDSS).

\begin{tabular}{cc}
\hline Escala de Severidade da Hiperidrose (HDSS) & Definição \\
\hline HDSS-1 & $\begin{array}{c}\text { Suor imperceptível } \\
\text { Não atrapalha atividades diárias }\end{array}$ \\
\hline HDSS-2 & $\begin{array}{c}\text { Suor tolerável } \\
\text { As vezes atrapalha as atividades diárias }\end{array}$ \\
\hline HDSS-3 & $\begin{array}{c}\text { Suor quase intolerável } \\
\text { HDSS-4 }\end{array}$ \\
\hline Frequentemente atrapalha atividades diárias \\
\hline Suor intolerável \\
\end{tabular}

Fonte: Lima SO, et al., 2020. Adaptado de Solish N, et al., 2007. 


\section{DISCUSSÃO}

O presente estudo mostrou uma prevalência estimada similar as de Augustin $\mathrm{M}$ et al. (2013) na Alemanha, Fujimoto T, et al. (2013) no Japão, Stefaniak T, et al. (2013) na Polónia, Lima SO, et al. (2015) e Lima SO, et al. (2019) no Brasil. Publicações mais antigas por Adar R, et al. (1997) em Israel e Strutton DR, et al. (2004) nos EUA mostraram um alcance diferente. Isso sugere que o aumento observado na prevalência de HP nas pesquisas recentes poderia ser explicado por um melhor conhecimento e uma melhor investigação da doença. $\mathrm{O}$ uso de uma abordagem de entrevista pessoal, realizado por pesquisadores clinicamente treinados no reconhecimento clínico de HP apresentou uma prevalência variando de $14,76 \%$ a $18 \%$. Portanto, diferentes métodos de investigação e melhor conhecimento médico podem ser responsáveis pela discrepância entre os dados de prevalência descritos mundialmente.

É importante ressaltar que a HP não parece depender da localização geográfica ou de grupos étnicos específicos. No Japão, a prevalência de HP por gênero foi de $16,66 \%$ entre os homens e $10,66 \%$ entre as mulheres (FUJIMOTO T, et al., 2013). Na Alemanha, variou de 18,1\% nos homens e $13,3 \%$ nas mulheres (AUGUSTIN M, et al., 2013).

$\mathrm{Na}$ Coreia do Sul, entre os indivíduos com diagnóstico de HP, 57,6\% eram homens (PARK EJ, et al., 2010). $\mathrm{Na}$ presente pesquisa, embora tenha havido maior disponibilidade pelo sexo feminino de responder ao presente questionário, não houve diferenças significativas entre os sexos quanto a presença de HP, semelhante aos resultados observados em outros estudos (TU Y-R, et al., 2007; FENILI R, et al., 2009; LIMA SO, 2015; LIMA SO e SANTANA VR, 2018; LIMA SO, et al., 2019). É uma doença crônica que produz desconforto e impactos negativos de maneira semelhante em ambos os sexos, verifica-se, no entanto que são as mulheres que mais procuram o tratamento da HP (FENILI R, et al., 2009; WESTPHAL FL, et al., 2011; FUJIMOTO T, et al., 2013; LIMA SO, et al., 2015; LIMA SO, et al., 2019).

A história da família foi presente para HP em 53\% dos casos em Israel, 17,9\% dos casos na China, 34,1\% dos casos na Coreia do Sul (TU Y-R, et al., 2007; PARK EJ, et al., 2010; STEFANIAK T, et al., 2013). Encontrase evidências que a hiperidrose primária tenha fator genético autossômico dominante com penetrância incompleta (KAUFMANN H, 2003). É possível que a HP seja um fator de risco para casais com um ou os dois cônjuges portador(es) transmitir(em) essa doença para sua prole. Na presente pesquisa, o caráter familiar foi relevante em $38,3 \%$ dos entrevistados. A maior divulgação do suor em excesso, sem causa conhecida ser uma doença, poderá melhorar a notificação da existência da HP em familiares dos portadores dessa afecção, reduzindo uma possível subnotificação.

Tu Y-R, et al. (2007) perceberam que o pico dos sintomas ocorre entre 6 e 16 anos. Na Coreia do Sul, a idade média de início foi de 15 anos (PARK EJ, et al., 2010). A idade de início dos entrevistados deste estudo era entre 0 a 19 anos e representou $82,5 \%$ dos entrevistados. As consequências negativas da doença incluem desconforto intenso e baixa autoestima para o paciente, afetando negativamente sua qualidade de vida em ambiente social, afetivo e profissional, fonte de piadas e bullying (BEATTIE PE e LEWIS-JONES MS, 2006).

Tratamentos clínicos ou cirúrgicos são sugeridos com bases científicas e devem ser considerados, o mais precoce possível com o intuito de minimizar as consequências dessa doença antes que a personalidade seja afetada. No entanto, a maioria dos pacientes procuram assistência médica apenas quando se tornam adultos, justificando o seu sofrimento durante a infância e a adolescência (BRAGANÇA GMG, et al., 2014). O diagnóstico precoce e o tratamento são necessários para melhorar a qualidade de vida, o que é especialmente importante nesse período de formação psicológica e da personalidade. O conhecimento dos pais sobre a HP ser uma doença crônica estimularia a procura do tratamento para seus filhos.

Strutton DR, et al. (2004), descreveu que a idade média dos pacientes diagnosticados com HP era 39,8 anos. Tu Y-R, et al. (2007) na China 17,6 anos, Park EJ, et al. (2010) na Coreia do Sul 28,5 anos, Delaplace M, et al. (2015) na França 29,3 anos, Fiorelli RKA, et al. (2011) no Brasil 25,4 anos. No presente estudo a idade média da presença de HP dos entrevistados foi de 25,9 anos. A faixa etária do portador, quando feito diagnóstico da HP, é um fator relevante, desde que essa é uma doença que tem início precoce e continua ativa na vida adulta. Esse agravo é impactante em um período da vida de desenvolvimento da vida pessoal e pico das atividades trabalhistas. 
A transpiração excessiva da HP, pode ser agravada por estímulos emocionais causando prejuízo nas atividades diárias e afetando negativamente a qualidade de vida (FIORELLI RKA et al., 2011; LESSA LR e FONTENELLE LF, 2011; BRAGANÇA GMG, et al., 2014; SOUZA LG, et al., 2015; LIMA SO, et al., 2019).

Bragança GMG, et al. (2013) em um estudo brasileiro mostrou que a HP, especialmente nas formas axilar e facial, era responsável por uma maior prevalência de ansiedade em relação à população geral e a pacientes com outras doenças crônicas. No presente estudo, $76,5 \%$ dos portadores referiram piora do suor excessivo sob estresse e $30,6 \%$ dos entrevistados afetados por HP relataram algum tipo de comprometimento nas atividades diárias.

Os profissionais que precisam manter um contato pessoa-a-pessoas para realizar seu trabalho podem parecer inseguros e/ou estressados em seu labor, dificultando a realização do mesmo. Isso leva a um aumento nos níveis de estresse e, em última análise, a um aumento da transpiração. A transpiração excessiva pode ocorrer principalmente nas regiões crânio-facial, palmar, axilar e plantar, isoladas ao associadas, causando ao seu portador situações constrangedoras que podem levar a introspecção e isolamento social.

Tu Y-R, et al. (2007), em um estudo com 13.000 estudantes de dez escolas de ensino médio e três faculdades, verificaram a prevalência de hiperidrose primária de 4,59\%, sem diferença entre os sexos, sendo a palmar isolada em $30,1 \%$ e a palmo-plantar em $42,18 \%$ dos portadores. As crianças com HP no âmbito escolar podem ser estigmatizadas como ansiosas tanto por parte de seus colegas como por seus educadores, por suarem em demasia. As mãos muitas vezes frias e molhadas estragam livros, cadernos e teclados de computador, para minimizar esses efeitos, utilizam constantemente toalhas ou enxugam as mãos na roupa.

Os meninos por andarem com toalha, seus colegas interrogam sua condição sexual. Na adolescência podem se tornar tímidos e evitam tocar a mão das outras pessoas. No âmbito doméstico, as vezes, não encontram o conforto esperado em virtude de seus familiares não valorizarem a sua doença (BELLET JS, 2010; KAUFFMAN P, 2018). Divulgar a existência da hiperidrose primária, tem o objetivo de melhorar 0 conhecimento desse agravo, permitindo a melhoria do relacionamento entre os pares no âmbito escolar e familiar

Lima SO, et al. (2019) verificaram que $18 \%$ dos acadêmicos de medicina de uma universidade privada, referiram ser portador de HP. Essa doença manifestava-se principalmente em sítios combinados como palmoplantar, sendo a região palmar a mais acometida, dificultando as funções acadêmicas. Na hiperidrose palmar, várias atividades laborais podem ser dificultadas, como manipulação de documentos importantes, desenhar projetos de arquitetura e engenharia, equilibrar ou manipular objetivos que possam escorregar das mãos como garçons.

Enfermeiros, dentistas, médicos, fisioterapeutas, fonoaudiólogos ou outras profissões que necessitem o contato manual são prejudicadas pela presença das mãos frias, úmidas e/ou molhadas. A manifestação desse agravo, causa prejuízos nas atividades diárias, pessoais e laborativas, com impacto negativo na qualidade de vida (CAMPOS JJG, et al., 2019; LIMA SO, et al., 2019).

Enquanto cidadãos têm dificuldades e por vezes impossibilitam de realizarem atividades que necessitam do reconhecimento das digitais, como no registro civil, título eleitoral. É importante que a HP seja melhor debatida, permitindo a divulgação da doença, orientando o diagnóstico e a abordagem terapêutica.

A hiperidrose axilar surge principalmente na puberdade com o aumento da produção dos hormônios sexuais, tendo uma prevalência em torno de $50,8 \%$ dos portadores de HP nessa faixa etária (STRUTTON DR, et al., 2004; KAUFFMAN P, 2018). Na HP axilar ocorre a formação de manchas de suor nas roupas, podendo estar associada a cromoidrose e a um odor fétido e desagradável, conhecido como bromidrose (SEMKOVA K, et al., 2015).

As roupas impregnadas por esse suor e odor, são precocemente descartadas e seus portadores referem que são estigmatizados por provocar a impressão de má higiene. Seus portadores evitam o uso de roupas coloridas, preferindo roupas brancas ou pretas, utilizam também truques tais como uso de absorventes axilares. A hiperidrose plantar geralmente está associada com a palmar e seu início ocorre na infância e adolescência, sendo acompanhada por bromidrose. 
Esta causa dificuldades no uso de sandália abertas que se tornam escorregadias acumulando poeira e estragando rapidamente os calçados. Seus portadores são obrigados a usar sapatos fechados com intuito de maior conforto e equilíbrio reduzindo escorregões, entorses e quedas. Este artifício, no entanto, causa o acúmulo de suor no sapato fechado, facilitando o aparecimento de micoses e aumentando o odor fétido. As mulheres têm dificuldade de usar salto alto dificultando o exercício de determinadas profissões como modelo e recepcionista.

$\mathrm{Na}$ hiperidrose crânio facial o gotejamento de suor da fronte e do couro cabeludo dá um aspecto de constante cansaço, além de dificultar o uso de maquiagem, protetor solar e outros cosméticos. Profissionais que necessitam de contato com o público por sua aparência ou proximidade se sentem prejudicados pela presença do suor, a exemplo de professores, aeromoças, recepcionistas e psicólogos (SANTANA VR, 2017; KAUFFMAN P, 2018; CAMPOS JJG, et al., 2019). Augustin M, et al. (2013) observaram que somente 27\% dos portadores de HP tinham consultados médicos e destes $28 \%$ utilizaram algum tipo de terapêutica. Lima $\mathrm{SO}$, et al. (2015) verificaram que entre estudantes de medicina portadores de HP, o diagnóstico dessa doença, foi dado por profissionais médico somente em $22,72 \%$. O diagnóstico da HP pode ser dado por critérios clínicos (FELINI R, et al., 2009), entretanto mesmo em portadores de HP da área de saúde houve uma baixa incidência do diagnóstico firmado por um médico.

É de grande relevância o conhecimento da HP como doença crônica, orientando aos pais da importância da procura de profissionais para tratamento, ainda na fase inicial da doença, minimizando os transtornos que o indivíduo passa no período infanto-juvenil. Alguns portadores da HP, mesmo na idade adulta, não tem o conhecimento de ser portador de uma doença que tem cura. Embora a HP seja reconhecida pelo código internacional de doenças (CID-10 R61.0), ainda é desconhecida por alguns profissionais, como educadores e da área de saúde, além de ter algumas vezes seu tratamento dificultado pelo sistema único de saúde. Para tanto o conhecimento da prevalência da hiperidrose primária é essencial, tanto para o conhecimento da população como de profissionais que as vezes desconhecem a existência e tratamento desta afecção (LIMA SO, et al., 2019).

A presente pesquisa reafirma o benefício do diagnóstico precoce e do tratamento desta doença crônica para minimizar ou evitar danos na qualidade de vida de pacientes com HP. O uso de entrevistas pessoais e individuais para avaliar a prevalência de HP conduzido por pesquisadores treinados para identificar HP, Médicos e Estudantes de Medicina deve aumentar a sensibilidade da pesquisa.

\section{CONCLUSÃO}

A prevalência de hiperidrose primária na população amostrada, avaliada aleatoriamente em cinco bairros, em uma capital do nordeste brasileiro, foi de $15,9 \%$, sendo semelhante em ambos os sexos e, na maioria dos casos, bilateral. O início dos sintomas ocorreu principalmente durante a infância e adolescência, com implicações negativas nas atividades diárias, e os sintomas primários de hiperidrose são exacerbados com o estresse. O diagnóstico precoce e o tratamento, é especialmente importante, nesse período de formação da personalidade. A história familiar foi relatada por mais de um terço dos entrevistados e por ser um fator genético autossômico dominante com penetrância incompleta, denota a possibilidade desse agravo ser transmitido para a prole de casais portadores de HP.

\section{REFERÊNCIAS}

1. ADAR R, et al. Palmar hyperhidrosis and its surgical treatment: a report of 100 cases. Ann Surg, 1977; $186(1): 34$.

2. AUGUSTIN M, et al. Prevalence and Disease Burden of Hyperhidrosis in the Adult Population. Dermatology, 2013; 227(1):10-3.

3. BEATTIE PE, LEWIS-JONES MS. A comparative study of impairment of quality of life in children with skin disease and children with other chronic childhood diseases. Br J Dermatol, 2006; 155(1):145-51.

4. BELLET JS. Diagnosis and Treatment of Primary Focal Hyperhidrosis in Children and Adolescents. Semin Cutan Med Surg, 2010; 29(2):121-6.

5. BRAGANÇA GMG, et al. Evaluation of anxiety and depression prevalence in patients with primary severe hyperhidrosis. An Bras Dermatol, 2014; 89(2):230-5.

6. CAMPOS JJG, et al. Impact of primary hyperhidrosis on the life of physical education academics of a university in the Brazilian Northeast. Motriz: rev educ fis, 2019; 25(2):e101914. 
7. CERFOLIO RJ, et al. The Society of Thoracic Surgeons Expert Consensus for the Surgical Treatment of Hyperhidrosis. Ann Thorac Surg, 2011;91(5):1642-8.

8. DEL SORBO F, et al. Primary Focal Hyperhidrosis in a New Family Not Linked to Known Loci. Dermatology, 2011; 223(4):335-42.

9. DELAPLACE M, et al. Factors associated with long-term outcome of endoscopic thoracic sympathectomy for palmar hyperhidrosis: a questionnaire survey in a cohort of French patients. Br J Dermatol, 2015; 172(3):805-7.

10. FELINI R, et al. Prevalência de hiperidrose em uma amostra populacional de Blumenau-SC, Brasil. Anais Brasileiros de Dermatologia, 2009; 84(4): 361-366.

11. FIORELLI RKA, et al. Avaliação do Impacto na Qualidade de Vida de Pacientes Portadores de Hiperidrose Primária Submetidos à Simpatectomia Videotoracoscópica. Rev Meta Aval. 2011; 3(7):1-24.

12. FUJIMOTO T, et al. Epidemiological study and considerations of primary focal 92 hyperhidrosis in Japan: From questionnaire analysis. J Dermatol, 2013; 40(11):886- 90.

13. GROSS KM, et al. Elevated Social Stress Levels and Depressive Symptoms in Primary Hyperhidrosis. Slominski AT, organizador. PLoS ONE, 2014; 9(3):e92412.

14. HEIDEMANN E, LICHT PB. A Comparative Study of Thoracoscopic Sympathicotomy Versus Local Surgical Treatment for Axillary Hyperhidrosis. Ann Thorac Surg, 2013; 95(1):264-8.

15. HONG C-HH, et al. Clinical Evaluation of a Microwave Device for Treating Axillary Hyperhidrosis: Dermatol Surg, 2012; 38(5):728-35.

16. HORNBERGER J, et al. Recognition, diagnosis, and treatment of primary focal hyperhidrosis. J Am Acad Dermatol. agosto de 2004; 51(2):274-86.

17. KAUFFMAN P. Primary Hyperhidrosis. In LOUREIRO M, CAMPOS J, WOLOSKER N, KAUFFMAN P, editors. Hyperhidrosis. Chan: Springer; 2018. p. 27-32.

18. KAUFMANN H, et al. Primary hyperhidrosis--evidence for autosomal dominant inheritance. Clin Auton Res. 2003; 13(2):96-8

19. LAKRAJ A-A, et al. Hyperhidrosis: Anatomy, Pathophysiology and Treatment with Emphasis on the Role of Botulinum Toxins. Toxins, 2013; 5(4):821-40.

20. LESSA LR, FONTENELLE LF. Botulinum toxin as a treatment for social phobia with hyperidrosis. Arch Clin Psychiatry, $2011 ; 38(2): 84-86$.

21. LIMA SO, et al. Infraareolar access for thoracoscopic sympathectomy to treat primary hyperhidrosis. Surg Today, 2013; 43(2): $221-4$.

22. LIMA SO, et al. Perfil Epidemiológico e Qualidade de Vida dos Estudantes de Medicina Portadores de Hiperidrose Primária. Revista Brasileira de Educação Médica, 2019; 43(1), 386-394.

23. LIMA SO, et al. Research of primary hyperhidrosis in students of medicine of the State of Sergipe, Brazil. An Bras Dermatol, 2015; 90(5):661-665.

24. LIMA SO, SANTANA VR. The prevalence of hyperhidrosis worldwide. In LOUREIRO M, CAMPOS J, WOLOSKER N, KAUFFMAN P, editors. Hyperhidrosis. Chan: Springer; 2018. p. 33-38.

25. MIOT HA. Sample size in clinical and experimental trials. J Vasc Bras, 2011; 10(4):275-278.

26. PARK EJ, et al. An Epidemiological Study of Hyperhidrosis Patients Visiting the Ajou University Hospital Hyperhidrosis Center in Korea. J Korean Med Sci, 2010; 25(5):772.

27. SANTANA VR. Prevalência de hiperidrose primária em grupos populacionais do estado de Sergipe, Brasil e Avaliação da qualidade de vida antes e após simpatectomia lombar para o tratamento de hiperidrose plantar. Tese (Doutorado em Saúde e Ambiente). Universidade Tiradentes, Aracaju, 2017; 153p.

28. SCHNEIER FR, et al. Social anxiety and functional impairment in patients seeking surgical evaluation for hyperhidrosis. Compr Psychiatry, 2012;53(8):1181-6.

29. SEMKOVA, K, et al. Hyperhidrosis, bromhidrosis, and chromhidrosis: Fold (intertriginous) dermatoses. Clin dermatol, 2015; 33(4):483-491.

30. SOLISH N, et al. A Comprehensive Approach to the Recognition, Diagnosis, and Severity-Based Treatment of Focal Hyperhidrosis: Recommendations of the Canadian Hyperhidrosis Advisory Committee. Dermatol Surg, 2007; 33(8):908-23.

31. SOUZA LG, et al. Radiofrequência bipolar no tratamento da hiperhidrose axilar: um estudo-piloto. Surg Cosmet Dermatol, 2015; 7(3): 228-231.

32. STEFANIAK T, et al. Is subjective hyperhidrosis assessment sufficient enough? Prevalence of hyperhidrosis among young Polish adults. J Dermatol, 2013; 40(10):819-823.

33. STRUTTON DR, et al. US prevalence of hyperhidrosis and impact on individuals with axillary hyperhidrosis: Results from a national survey. J Am Acad Dermatol, 2004; 51(2):241-8.

34. TU Y-R, et al. Epidemiological survey of primary palmar hyperhidrosis in adolescent in Fuzhou of People's Republic of China. Eur J Cardiothorac Surg, 2007; 31(4):737-9.

35. VORKAMP T, et al. Hyperhidrosis: Evolving concepts and a comprehensive review. The Surgeon, 2010; 8(5):287-92.

36. WALLING HW. Clinical differentiation of primary from secondary hyperhidrosis. J Am Acad Dermatol, 2011;64(4):6905.

37. WESTPHAL FL, et al. Prevalência de hiperidrose entre estudantes de medicina. Rev Col Bras Cir, 2011; 38(6):392397

38. WOLOSKER N, et al. Evaluation of quality of life over time among 453 patients with hyperhidrosis submitted to endoscopic thoracic sympathectomy. J Vasc Surg, 2012; 55(1):154-6.

39. WOLOSKER N, et al. The use of oxybutynin for treating facial hyperhidrosis. An Bras Dermatol, 2011; 86(3):451-456. 